\title{
Epidemiology of Cancer of Ovary : Changing Trends
}

\section{KISHORE CHAUDHRY}

Objective: To understand the changes in incidence rates of ovarian cancer over the years and study its geographic variations.

Methodology: Published literature from pubmed and data from cancer registry reports from various sources were assessed.

Magnitude: Ovarian cancer is the sixth commonest cancer among women globally and accounting for about 204,500 incident cases and about 125,000 deaths in 2002. About 21,150 women developed this disease and about 16,300 women died of ovarian cancer in India in 2002. More developed countries show a higher incidence of ovarian cancer as compared to less developed countries. Different countries have experienced different trends during the last two decades, with USA witnessing a decrease in incidence rate of ovarian cancer and England and Wales revealed an increase followed by a plateau. Population based cancer registries functioning under the National Cancer Registry Programme of ICMR show the annual incidence rate of ovarian cancer to vary between 1.3 and 7.6 per 100,000 women. It is among the top 5 cancer sites among women in most registries. The incidence rate seems to be higher in urban areas. Registries at Delhi and Mumbai have shown an increase in incidence rate of ovarian cancer over time. Most of the cases are detected when the disease has spread beyond ovary. The study of age specific rates over time in Mumbai shows that the rates increased in all age groups. Data from hospital based cancer registries in India shows that only a small proportion of cases are diagnosed at localized stage.

Risk Factors: Exact cause of ovarian cancer is not known. Major known risk factors of ovarian cancer are, low parity/ nulliparity, higher age at first pregnancy, certain genes (BRCA1 and BRCA2), women with a personal history of breast cancer or a family history of breast or ovarian cancer, and older women.

Prognosis: A small increase in survival rates has been observed during the last 2 to 3 decades in some countries. This has been attributed mainly to availability of platinum based chemotherapy. Although the survival of women with localized disease is excellent, evidence is not sufficient to suggest efficacy of currently known tools in their use as screening tools. 